\title{
A high-throughput pipeline for detecting locus-specific polymorphism in hexaploid wheat (Triticum aestivum L.)
}

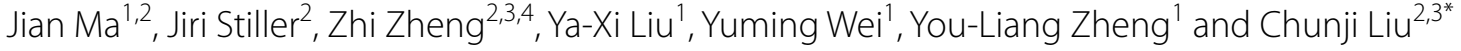

\begin{abstract}
Background: Bread wheat (Triticum aestivum L., $2 n=6 x=42$ ) is an allohexaploid with a huge genome. Due to the presence of extensive homoeologs and paralogs, generating locus-specific sequences can be challenging, especially when a large number of sequences are required. Traditional methods of generating locus-specific sequences are rather strenuous and time-consuming if large numbers of sequences are to be handled.

Results: To improve the efficiency of isolating sequences for targeted loci, a time-saving and high-throughput pipeline integrating orthologous sequence alignment, genomic sequence retrieving, and multiple sequence alignment was developed. This pipeline was successfully employed in retrieving and aligning homoeologous sequences and 83\% of the primers designed based on the pipeline successfully amplified fragments from the targeted subgenomes.

Conclusions: The high-throughput pipeline developed in this study makes it feasible to efficiently identify locus-specific sequences for large numbers of sequences. It could find applications in all research projects where locus-specific sequences are required. In addition to generating locus-specific markers, the pipeline was also used in our laboratory to identify differentially expressed genes among the three subgenomes of bread wheat. Importantly, the pipeline is not only valuable for research in wheat but should also be applicable to other allopolyploid species.
\end{abstract}

Keywords: Allopolyploid, Multiple sequence alignment, blastn, Primer design, Genome-specificity, Sequence polymorphism

\section{Background}

Reference genome sequences of several major crops have been reported and include rice [1], barley [2], foxtail millet [3], maize [4], sorghum [5], potato [6], tomato [7] and Brassica napus [8]. Significant progress has also been made in recent years in generating reference genomes for bread wheat [9] and its progenitor species [10, 11]. These genome sequences have been extensively exploited in the whole spectrum of biological studies ranging from basic understanding of crop evolution to applied breeding. With the rapid development in sequencing capacity, it is anticipated that whole genome sequences should soon

\footnotetext{
*Correspondence: chunji.liu@csiro.au

${ }^{3}$ School of Plant Biology, The University of Western Australia, Perth, WA 6009, Australia

Full list of author information is available at the end of the article
}

become available for multiple genotypes for each of the species of agronomic importance.

Knowing the origins of specific DNA or RNA sequences is essential in numerous applications, such as designing locus-specific markers. Although gene duplication is a common feature of all plant species including Brachypodium [12], rice [1], and barley [2], developing locus-specific markers for these diploid species is relatively easy. This suggests that enough variation must exist between the majority of duplicated genes in these species. However, isolating locus-specific sequences for a given subgenome of interest from bread wheat or other polyploid species is still challenging as two or more homoeologous sequences exist in each of these genomes. It can be even more daunting when isolating a gene of interest which belongs to an orthologous gene set or a gene family. 
Currently several steps need to be taken when isolating a specific homoeologous sequence from an allopolyploid species. First, web-based blast servers such as National Center for Biotechnology Information (NCBI) or ViroBLAST in Unité de Recherche Génomique Info (URGI, https://urgi.versailles.inra.fr/blast/blast.php) [13] need to be employed to search for orthologous sequences for a given query sequence. Second, all the orthologous gene sequences for a given species need to be manually retrieved from contigs or scaffold sequences. Third, multiple sequence alignment tools are required to align retrieved orthologous sequences to detect locus-specific sequences. This procedure can be used to manage a limited number of sequences but will become rather strenuous and time-consuming if large numbers of sequences need to be handled.

To improve the efficiency of retrieving sequences from polyploidy species, we have developed a pipeline by streamlining the steps in orthologous sequence alignment, genomic sequence retrieving and multiple

Table 1 Numbers of genes used in blasting against wheat chromosome shotgun sequences (CSSs)

\begin{tabular}{llll}
\hline & Brachypodium distachyon & Triticum urartu & Aegilops tauschii \\
\hline Total numbers of genes & 31,029 & 34,879 & 43,150 \\
Genes with hits on CSSS & 30,028 & 34,671 & 43,126 \\
Genes with generated alignments & 27,782 & 32,378 & 40,961 \\
\hline
\end{tabular}

\section{a}

Chr1AL 3874570 Chr1BL 3754956

Chr1AL 3874570 Chr1BL 3754956

Chr1AL_3874570 Chr1BL 3754956

\section{C}

Chr1DL_2247910_ Chr1AL 3901045 Chr1BL_3905741_ Chr1DL 2228030 Chr1AL_3887755

Chr1DL_2247910_ Chr1AL_3901045 Chr1BL 3905741 Chr 1DL_2228030_ Chr 1 AL_3887755

Chr1DL 2247910 Chr1AL_3901045 Chr1BL 3905741 Chr 1DL_2228030_ Chr1AL_3887755

$190 \quad 500 \quad 510 \quad 520 \quad 530 \quad 510$

\section{b}

Chr1BL_3915727 Chr1AL_3911108_ Chr1DL_2273708

ACAGGAGCGGCCTTGCCGTCCCCGGCGAGCGGCACGGCGGCGCCGGAGGCAACCACACCA ACAGGAGCGGCCTCGCCGTCCCCGGCGAGCGGCACGGCGGCGCCGGCGGCAACCACACCA 570

580

59

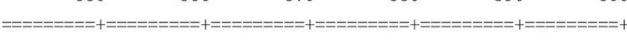
CCCAGGTAAGCTGAGCTTCCCGGACGAGCACCTGACAGCCGTGAGCGGCCGGTACGGCGC CCCAGGTAAGCTGAGCTTCCCGGACGAGCACCTGACGGCCGTGAGCGGCCGCTACGGCGC

\begin{tabular}{|c|c|c|c|c|c|}
\hline 610 & 620 & 630 & 640 & 650 & 660 \\
\hline
\end{tabular}

GGTGGCGCCCGGCGGCTCGCCGGTGATCCGGTCGCTGGCGTTCAGGACGGAGCGCGCGGC GGTGGCTCCCGGTGGCTCGCCAGTGATCCGGTCGCTGGCGTTCAGGACGGAGCGCGCGGC

Fig. 1 Examples of multiple alignments for two (a), three (b), and five (c) homoeologous sequences for identifying allele-specific sequences for marker development. 


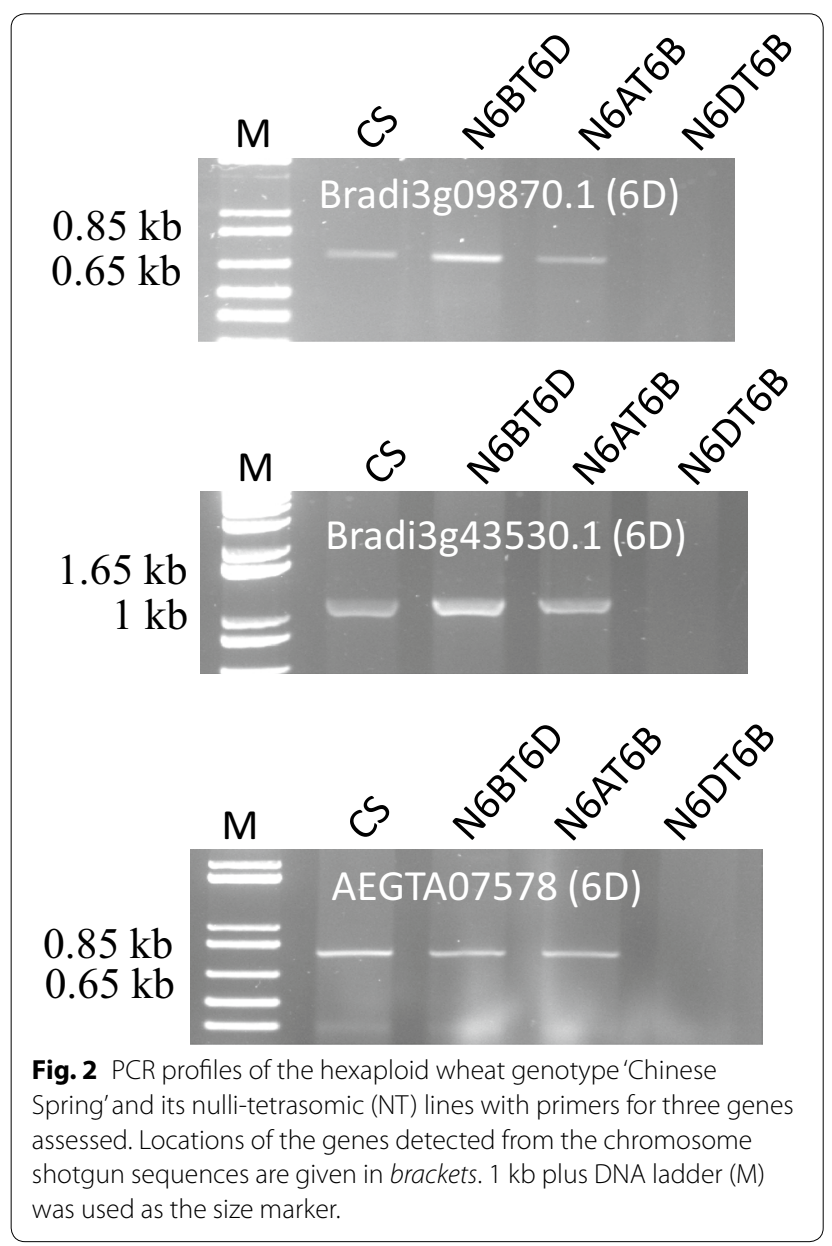

sequence alignment. This time-saving and high-throughput pipeline significantly simplifies the detection of locus-specific sequences in allopolyploid species. The pipeline has also been successfully used in differentiating expressed genes among the three bread wheat subgenomes.

\section{Results and discussion}

The percentages of query gene sequences which detected two or more orthologous sequences from chromosome shotgun sequences (CSSs) were about 90\% from Brachypodium, 93\% from Ae. tauschii and 95\% from T. urartu (Table 1). Examples of these stringent alignments containing two orthologous sequences (e.g. Bradi2g16370.1), three homoelogous sequences (e.g. Bradi2g33190.1), and more than four homoeologous sequences (e.g. Bradi2g14840.1) are shown in Fig. 1. Each of the alignments with the suffix '.htm' is easily readable by any web browser. The alignments generated and described in 'Methods' can be directly used to check possible allele-specific loci for isolating genes in hexaploid wheat.
Given that primers designed based on a single nucleotide polymorphisms (SNP) did not always amplify a specific fragment in our previous studies, primers designed in this study were based on two or more SNPs or indels (Additional file 1: Table S1). Of the 36 primer pairs designed for selected loci, $30(83 \%)$ amplified a product on the expected chromosomes, two failed to amplify any PCR products, and the other four generated locusspecific fragments (Fig. 2 and Additional file 1: Table S1). Eleven of the 30 pairs of primers were further assessed against other bread wheat genotypes (Additional file 1: Table S1). Sequence alignments indicated that, without exception, they all amplified sequences homologous with those from the expected chromosomes as shown in 'Chinese Spring' ('CS') (Additional file 1: Table S1). Four of these primer pairs generated polymorphic fragments between the parents of the mapping population used in this study. The polymorphic sequences were used to develop cleaved amplified polymorphic sequence (CAPS) markers. Each of the four CAPS markers was successfully mapped to the anticipated chromosome as originally detected using 'CS' aneuploids (Fig. 3, Additional file 2: Fig. S1 and Additional file 3: Fig. S2).

A pipeline for generating SNP markers, PolyMarker, was reported recently and it is used to design primers for KASP $^{\mathrm{TM}}$ (Kompetitive Allele Specific PCR) assay. KASP ${ }^{\mathrm{TM}}$ is a very unique system in that it uses three primers in PCR reactions. Two of them are allele-specific forward primers. Sequences from parental genotypes are required in designing the two forward primers which make accurate bi-allelic discrimination possible [14-18].

Different from the PolyMarker/KASPTM system, the method reported in this paper does not need sequences from parental genotypes. Allele-specific primers are designed based on sequence alignments from all subgenomes of a species. In addition to designing allele-specific markers as shown in the current study, we have also adapted the pipeline to design allele-specific primers for reverse transcription quantitative PCR (RT-qPCR) analysis in wheat. For example, several positions of orthologous sequences of Bradilg04060 could be used to design RT-qPCR primers in bread wheat (Fig. 4). We have also successfully used the pipeline to retrieve conserved regions that could be used for differentially expressed analysis in bread wheat (not published). Obviously, this high-throughput pipeline would be applicable to other allopolyploid species such as rapeseed, cotton, or oat.

\section{Conclusion}

Here we reported on a high-throughput pipeline which integrates orthologous sequence alignment, genomic sequences retrieval, and multiple sequence alignment. The pipeline can be conveniently used to identify 


\section{a}

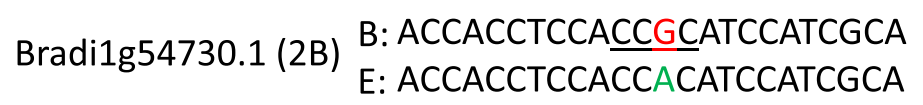

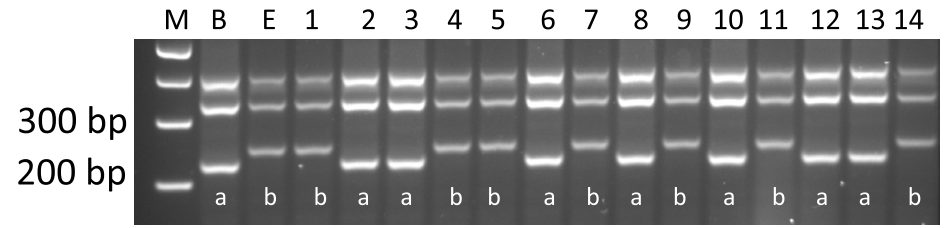

b

\section{Bradi1g55847.1(2B) B: GGGGTGGGCGGCCGTGTTCTTGC
E: GGGGTGGGCGGCTGTGTTCTTGC}

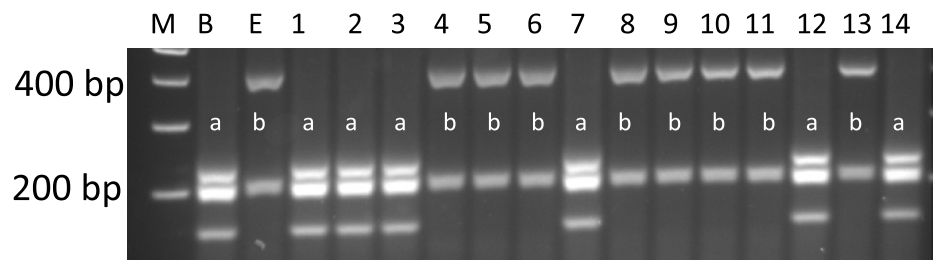

C

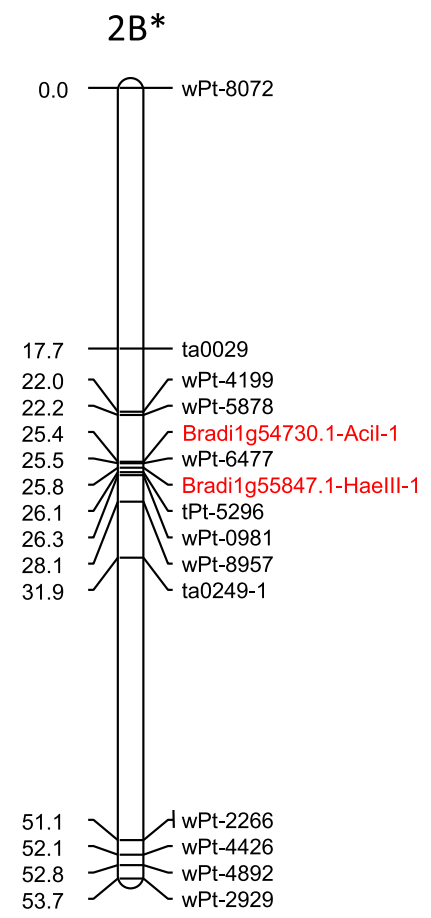

Fig. 3 Validation of marker locations using a DH (doubled haploid) population. Orthologous sequences of Bradi1g54730.1 and Bradi1g55847.1 were amplified from the two parents of the DH population, B ('Batavia') and E ('Ernie'), and sequenced. The single nucleotide polymorphism (in red and green) and restriction enzyme sites (underlined) were identified between B and E for Bradi1g54730.1 with restriction enzyme Acil (a) and Bradilg55847.1 with Haell $(\mathbf{b})$. The amplified products of the two parents and 14 of the DH lines were digested and separated on agarose gels. Map positions of the two new markers on chromosome 2B (c) were calculated based on the linkage map published by Li et al. [21].

locus-specific sequences for marker development and RT-qPCR and transcriptome analyses, especially when large numbers of sequences need to be dealt with. Examples of its application in wheat are given in this publication but the pipeline would also be valuable for similar applications in other allopolyploid species as well.

\section{Methods}

\section{Plant materials}

The euploid and selected nullisomic-tetrasomic 'CS' lines $[19,20]$ were used to locate PCR-amplified fragments to specific chromosomes. Two wheat populations were employed to further validate the location of DNA fragments amplified from primers designed in this study. One is a doubled haploid (DH) population with 153 lines generated from the 'Batavia'/'Ernie' cross [21], and the other one is an F8 population of recombinant inbred lines (RILs) with 92 lines derived from the 'Lang'/'CSCR6' cross [22].

\section{Data collection}

Gene-coding sequences (CDS) of Brachypodium genome version 1.2 were downloaded from http:// www.plantgdb.org/BdGDB [12]. CDS of Ae. tauschii (wheatD_final_43150.gff.cds) [10] and T. urartu (TRIUR3_120813_filter150_cds) [11] were both downloaded from GIGA_DB (http://gigadb.org/). CSSs of 'CS' were downloaded from https://urgi.versailles.inra.fr/ download/iwgsc/Science/ [9].

\section{Generation of multiple sequence alignments and primer design}

Alignments of orthologous sequences from Brachypodium, Ae. tauschii, and T. urartu were generated following the steps outlined in Fig. 5. First, gene sequences from Brachypodium, Ae. tauschii, and T. urartu were blasted against CSSs using the BLAST + blastn algorithm with the parameters '-num_descriptions 10 -num_ alignments 10 -evalue 0.00001 ' (i.e. a maximum of 10 hits 


\section{Bradi1g04060. 1}

Chr5BL_10882237

Chr5AL_2690175_

Chr5DL_4565250

Chr5BL_10882237

Chr5AL_2690175

Chr5DL_4565250

Chr5BL_10882237

Chr5AL_2690175

Chr5DL_4565250

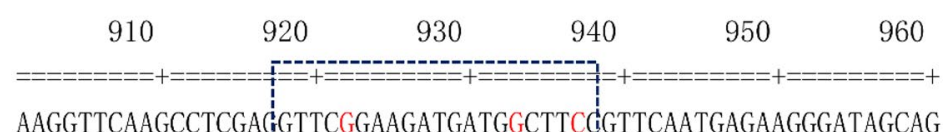
AAGGTTCAAGCCTCGAGGTTCGGGAGATGATGACTTCGGTTCAATGAGAAGGGATAGCAG AAGGTTCAAGCCTCGAGGTTCAGAAGATGATGGCTTTGGTCAATGAGAAGGGATAGCAG

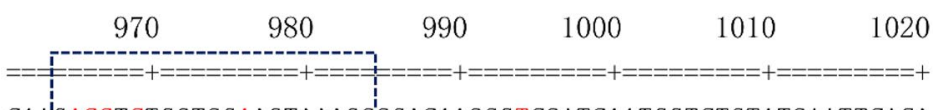
CAAGACCTCTGGTGGAAGTAAAGGGGACAAGGGTCGATCAATGGTCTGTATGAATTCACA CAAGGTTTATGGTGGGAGTAAAGGGGACAAGGGTCGATCAATGGTCTGTATGAATTCACA CAAGACCTCTGGTGGAAGTAAAGGGACAAGGGACGATCAATGGTCTGTATGAATTCACA

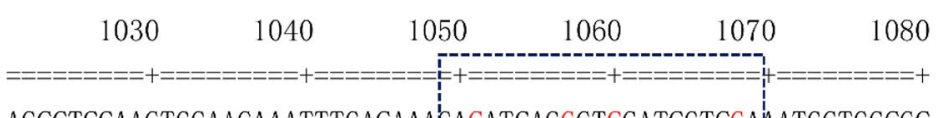
AGCCTCCAAGTGGAAGAAATTTGACAAAGACATCAGGGTCGATCGTCGAHATGGTGGCGC AGCCTCCAAGTGGAAGAAATTTGACAAAGACATCAGGGTCGATCGTCAAAATGGTGGCAC AGCCTCCAAGTGGAAGAAATTTGACAAA'GATATCAGAGTTGATCGTCGAAATG

$\begin{array}{llllll}1090 & 1100 & 1110 & 1120 & 1130 & 1140\end{array}$ Chr5BL_10882237 Chr5AL_2690175 Chr5DL_4565250

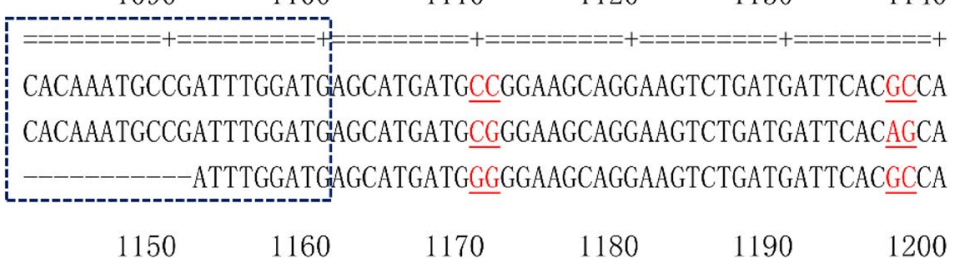

Chr5BL_10882237

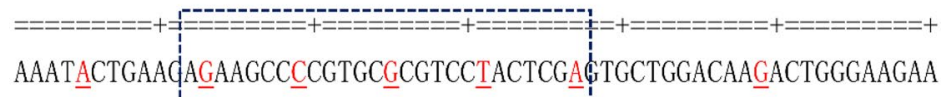

Chr5AL_2690175

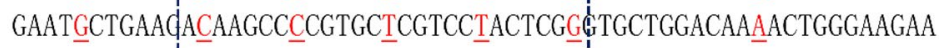

Chr5DL_4565250 GAATACTGAAdAGAAGCCTCGTGCTCGTCCCACTCGGGTGCTGGACAAGACTGGGAAGAA

1210 1220 1230

1240

1250

1260

Chr5BL_10882237

Chr5AL_2690175

Chr5DL_4565250

ACTCAGGGTCTATAAGAAGGATTCAGTTTCTGATTCTGAGGAAATTGCTCCTCCTAAGAA ACTCAGGGTCTATAGGAAGGATTCAGTTTCTGACTCTGAGGAAATTGCTCCTCCTAAGAA ACTCAGGGTCTATAAGAAGGATTCAGTTTCTGACTCTGAGGAAATTGCTCCTCCGAAGAA

Fig. 4 An example of selecting primer sequences for qPCR analysis. Dotted boxes represent regions that could be used for primer design.

for each gene query and with E-value threshold of $10^{-5}$ ) [23]. Second, an in-house script was used to retrieve the coordinates of each hit for a given gene query from the blast results. A maximum of 5,000 bp intron and minimum of $200 \mathrm{bp}$ exon were used to limit the retrieved coordinates for a given hit. Third, the $5^{\prime}$ and $3^{\prime}$ flanking regions of $300 \mathrm{bp}$ were isolated from each of the contigs (hits) according to the coordinates obtained. Fourth, the isolated genomic sequences from all the hits for a given query were written to a single file. Finally, a script integrated with Gblocks_0.91ba [24] and Clustal W 2.1 [25] was used to generate the alignments of all the retrieved genomic sequences for a given query (Fig. 5). The alignments and in-house developed scripts are available at http://dx.doi.org/10.6084/m9.figshare.1393103; http:// dx.doi.org/10.6084/m9.figshare.1393106; http://dx.doi. org/10.6084/m9.figshare.1393105.

\section{Validation of primers designed from the alignments}

For validating the efficiency of the multiple sequence alignments generated, genes that were polymorphic between the parental lines of the mapping populations 


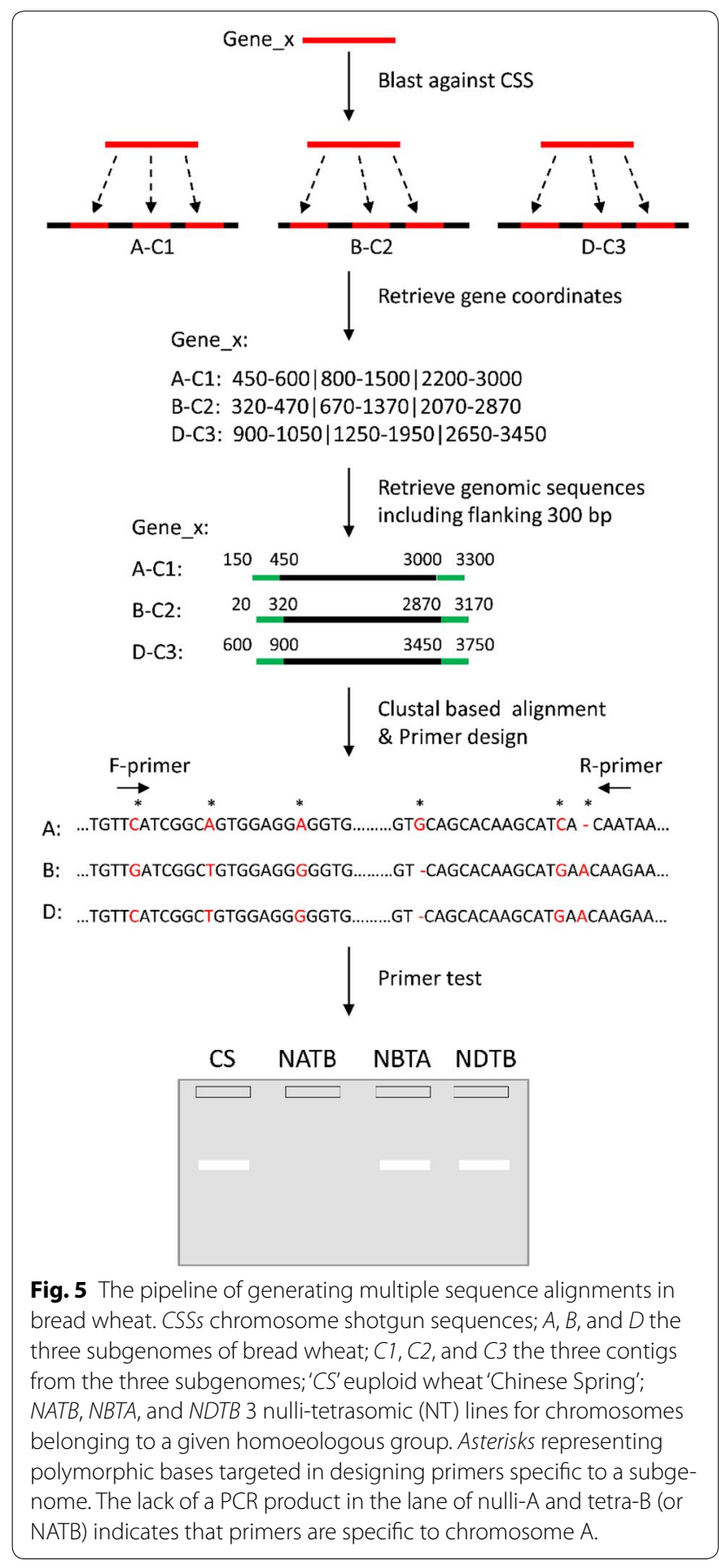

were assessed. Where possible, sequences differing in more than 1 SNP or indel were used to design primers targeting specific chromosomes for the selected genes (Additional file 1: Table S1).

The euploid and selected nullisomic-tetrasomic lines of 'CS' $[19,20]$ were analysed. Genomic DNA was extracted from 20-day-old seedlings using the hexadecyltrimethylammonium bromide method
(CTAB) [26]. PCR amplification was performed in $10 \mu \mathrm{l}$ reaction mixtures with $50 \mathrm{ng}$ of genomic DNA, $200 \mu \mathrm{M}$ of each dNTP, $0.2 \mu \mathrm{M}$ of each primer, and 0.5 units of Taq polymerase. The cycling parameters were $94^{\circ} \mathrm{C}$ for $5 \mathrm{~min}$ to pre-denature, which was followed by 35 cycles of $94^{\circ} \mathrm{C}$ for $45 \mathrm{~s}, 40 \mathrm{~s}$ at the appropriate annealing temperature (ranging from 50 to $70^{\circ} \mathrm{C}$ depending on the primers, see Additional file 1: Table $\mathrm{S} 1), 72^{\circ} \mathrm{C}$ for $1 \mathrm{~min}$, and a final extension at $72^{\circ} \mathrm{C}$ for $10 \mathrm{~min}$. Amplification products were separated on $1.5 \%$ agarose gels.

To further confirm the effectiveness of the primers in the RIL and DH populations, fragments of interest were purified using the QIAquick Extraction Kit (QIAGEN). The recovered PCR fragments were inserted into the pGEM-T easy vector (Promega) and transformed into Escherichia coli (Top10). At least three independent clones for each fragment were sequenced in both directions by the Australian Genome Research Facility Ltd. Sequenced fragments were aligned using by the DNAman software package (V5. 2.10; Lynnon Biosoft). To identify whether the sequenced fragments were from the expected chromosomes as found in the 'CS' aneuploids, they were aligned with all of the orthologous sequences from 'CS' for a given gene. SNPs between the parents of a given population were exploited to develop cleaved amplified polymorphic sequence (CAPS) marker using dCAPS Finder 2.0 [27]. PCR products were digested with appropriate enzymes from New England Biolabs (NEB) based on target sequences differences and separated on $3 \%$ agarose gels. The genetic linkage map was generated using JoinMap 4 [28].

\section{Additional files}

Additional file 1: Table S1. Details of primers tested.

Additional file 2: Figure S1. Validation of marker location of AEGTA18760 using a RIL (recombination inbred lines) population. Orthologous sequences of AEGTA18760 were amplified from the two parents of the RIL population, C ('CSCR6') and L ('Lang'), and sequenced. The single nucleotide polymorphism (in red and green) and restriction enzyme sites (underlined) were identified between $C$ and $L$ for AEGTA18760 with restriction enzyme N/alll (A). The amplified products of the two parents and 13 of the RIL lines were digested and separated on agarose gels. The map position of the new marker on chromosome 3B (B) was calculated based on the linkage map published by Ma et al. [22].

Additional file 3: Figure S2. Validation of marker location of Bradilg07500.1 using a DH (doubled haploid) population. Orthologous sequences of Bradilg07500.1 were amplified from the two parents of the $\mathrm{DH}$ population, B ('Batavia') and E ('Ernie'), and sequenced. The single nucleotide polymorphism (in red and green) and restriction enzyme sites (underlined) were identified between B and E for Bradilg07500.1 with restriction enzyme Btgl (A). The amplified products of the two parents and 13 of the RIL lines were digested and separated on agarose gels. The map position of the new marker on chromosome 3B (B) was calculated based on the linkage map published by Li et al. [21]. 


\section{Abbreviations}

CS: Chinese spring; DH: doubled haploid; RILs: recombinant inbred lines; CDS coding sequences; CSSs: chromosome shotgun sequences; CAPS: cleaved amplified polymorphic sequence; CTAB: hexadecyltrimethylammonium bromide; SNP: single nucleotide polymorphism; RT-qPCR: reverse transcription quantitative PCR; NCBI: National Center for Biotechnology Information.

\section{Authors' contributions}

Conceived and designed the experiments: CL JM JS WM Y-LZ. Performed the experiments: JM JS ZZ Y-XL. Analysed the data and wrote the paper: JM JS Y-LZ $\mathrm{CL}$. All authors read and approved the final manuscript.

\section{Author details \\ ${ }^{1}$ Triticeae Research Institute, Sichuan Agricultural University, Wenjiang, Chengdu 611130, China. ${ }^{2}$ CSIRO Agriculture Flagship, 306 Carmody Road, St Lucia, QLD 4067, Australia. ${ }^{3}$ School of Plant Biology, The University of Western Australia, Perth, WA 6009, Australia. ${ }^{4}$ National Foxtail Millet Improvement Centre, Institute of Millet Crops, Hebei Academy of Agricultural and Forestry Sciences, Shijiazhuang, China.}

\section{Acknowledgements}

This publication is based upon work supported by a project from the International Science \& Cooperation Program of China (No. 2015DFA30600). JM is grateful to the Sichuan Agricultural University and the China Scholarship Council for funding his visit to CSIRO Agriculture Flagship. The authors are grateful to Drs Evans Lagudah (CSIRO) and Peng Zhang (Plant Breeding Institute, The University of Sydney) for providing the aneuploid stocks of 'Chinese Spring', and to Drs Cushla Metcalfe and Jason Carere for helping to improve the manuscript.

\section{Compliance with ethical guidelines}

\section{Competing interests}

The authors declare that they have no competing interests.

Received: 15 May 2015 Accepted: 22 July 2015

Published online: 04 August 2015

\section{References}

1. Yu J, Hu SN, Wang J, Wong GKS, Li SG, Liu B et al (2002) A draft sequence of the rice genome (Oryza sativa L. ssp indica). Science 296(5565):79-92. doi:10.1126/science.1068037

2. International-Barley-Genome-Sequencing-Consortium (2012) A physical, genetic and functional sequence assembly of the barley genome. Nature 491(7426):711-716

3. Zhang G, Liu X, Quan Z, Cheng S, Xu X, Pan S et al (2012) Genome sequence of foxtail millet (Setaria italica) provides insights into grass evolution and biofuel potential. Nat Biotechnol 30(6):549-554

4. Schnable PS, Ware D, Fulton RS, Stein JC, Wei F, Pasternak S et al (2009) The B73 maize genome: complexity, diversity, and dynamics. Science 326(5956):1112-1115

5. Paterson AH, Bowers JE, Bruggmann R, Dubchak I, Grimwood J, Gundlach $\mathrm{H}$ et al (2009) The Sorghum bicolor genome and the diversification of grasses. Nature 457(7229):551-556

6. Consortium PGS (2011) Genome sequence and analysis of the tuber crop potato. Nature 475(7355):189-195

7. Consortium TG (2012) The tomato genome sequence provides insights into fleshy fruit evolution. Nature 485(7400):635-641

8. Chalhoub B, Denoeud F, Liu S, Parkin IA, Tang H, Wang X et al (2014) Early allopolyploid evolution in the post-Neolithic Brassica napus oilseed genome. Science 345(6199):950-953

9. International-Wheat-Genome-Sequencing-Consortium (2014) A chromosome-based draft sequence of the hexaploid bread wheat (Triticum aestivum) genome. Science 345:1251788

10. Jia J, Zhao S, Kong X, Li Y, Zhao G, He W et al (2013) Aegilops tauschii draft genome sequence reveals a gene repertoire for wheat adaptation. Nature 496:91-95
11. Ling H-Q, Zhao S, Liu D, Wang J, Sun H, Zhang C et al (2013) Draft genome of the wheat A-genome progenitor Triticum urartu. Nature 496:87-90

12. Vogel JP, Garvin DF, Mockler TC, Schmutz J, Rokhsar D, Bevan MW et al (2010) Genome sequencing and analysis of the model grass Brachypodium distachyon. Nature 463(7282):763-768

13. Deng W, Nickle DC, Learn GH, Maust B, Mullins JI (2007) ViroBLAST: a stand-alone BLAST web server for flexible queries of multiple databases and user's datasets. Bioinformatics 23(17):2334-2336. doi:10.1093/ bioinformatics/btm331

14. Ramirez-Gonzalez RH, Uauy C, Caccamo M (2015) PolyMarker: a fast polyploid primer design pipeline. Bioinformatics 31(12):2038-2039

15. Ramirez-Gonzalez RH, Segovia V, Bird N, Fenwick P, Holdgate S, Berry S et al (2014) RNA-Seq bulked segregant analysis enables the identification of high-resolution genetic markers for breeding in hexaploid wheat. Plant Biotechnol J 13(5):613-624

16. Genomics L (2013) http://www.lgcgroup.com/services/genotyping. Accessed 30 July 2015

17. Kumpatla SP, Buyyarapu R, Abdurakhmonov IY, Mammadov JA (2012) Genomics-assisted plant breeding in the 21st century: technological advances and Progress. In: Abdurakhmonov I (ed) Plant breeding. InTech publishers

18. Semagn K, Babu R, Hearne S, Olsen M (2014) Single nucleotide polymorphism genotyping using Kompetitive Allele Specific PCR (KASP): overview of the technology and its application in crop improvement. Mol Breed 33(1):1-14

19. Sears E (1954) The aneuploids of common wheat. Univ Mo Agric Exp Stn Res Bull 572:1-58

20. Sears ER, Riley R, Lewis KR (1966) Nullisomic-tetrasomic combinations in hexaploid wheat. In: Riley R, Lewis KR (eds) Chromosome manipulation and plant genetics. Oliver and Boyd, London, pp 29-45

21. Li HB, Xie GQ, Ma J, Liu GR, Wen SM, Ban T et al (2010) Genetic relationships between resistances to Fusarium head blight and crown rot in bread wheat (Triticum aestivum L.). Theor Appl Genet 121(5):941-950

22. Ma J, Li H, Zhang C, Yang X, Liu Y, Yan G et al (2010) Identification and validation of a major QTL conferring crown rot resistance in hexaploid wheat. Theor Appl Genet 120(6):1119-1128

23. Altschul SF, Madden TL, Schaffer AA, Zhang JH, Zhang Z, Miller W et al (1997) Gapped BLAST and PSI-BLAST: a new generation of protein database search programs. Nucleic Acids Res 25(17):3389-3402. doi:10.1093/ nar/25.17.3389

24. Talavera G, Castresana J (2007) Improvement of phylogenies after removing divergent and ambiguously aligned blocks from protein sequence alignments. Syst Biol 56(4):564-577

25. Larkin MA, Blackshields G, Brown NP, Chenna R, McGettigan PA, McWilliam H et al (2007) Clustal W and clustal X version 2.0. Bioinformatics 23(21):2947-2948. doi:10.1093/bioinformatics/btm404

26. Murray M, Thompson WF (1980) Rapid isolation of high molecular weight plant DNA. Nucleic Acids Res 8(19):4321-4326

27. Neff MM, Turk E, Kalishman M (2002) Web-based primer design for single nucleotide polymorphism analysis. Trends Genet 18(12):613-615

28. Van Ooijen J (2006) Software for the calculation of genetic linkage maps (Kyazma BV, Wageningen, The Netherlands) version 4

\section{Submit your next manuscript to BioMed Central and take full advantage of:}

- Convenient online submission

- Thorough peer review

- No space constraints or color figure charges

- Immediate publication on acceptance

- Inclusion in PubMed, CAS, Scopus and Google Scholar

- Research which is freely available for redistribution

Submit your manuscript at www.biomedcentral.com/submit
C Biomed Central 